\title{
Variabilité de l'érosion actuelle et holocène : le cas des marbres de Grenville en Outaouais québécois
}

The Variability of Present-Day and Holocene Erosion within Grenville Marbles on the Québec Side of the Ottawa River Variabilität der Erosion in der Gegenwart und Im Holozän: Der Fall des Marmors von Grenville im Quebeker Outaouais

\section{Clément Prévost et Bernard Lauriol}

Volume 48, numéro 3, 1994

Les travertins

Travertines

URI : https://id.erudit.org/iderudit/033010ar

DOI : https://doi.org/10.7202/033010ar

\section{Aller au sommaire du numéro}

\section{Éditeur(s)}

Les Presses de l'Université de Montréal

ISSN

0705-7199 (imprimé)

1492-143X (numérique)

Découvrir la revue

Citer cet article

Prévost, C. \& Lauriol, B. (1994). Variabilité de l'érosion actuelle et holocène : le cas des marbres de Grenville en Outaouais québécois. Géographie physique et Quaternaire, 48(3), 297-304. https://doi.org/10.7202/033010ar
Résumé de l'article

Cet article souligne la difficulté de comparer les taux d'érosion mesurés dans les marbres de Grenville selon différentes méthodes. La variabilité des taux d'érosion a été mise en évidence par l'étude des nodules en relief, des plaquettes de calcaire et par l'hydrochimie. La mesure moyenne du déchaussement postglaciaire des nodules emprisonnés dans les marbres se situe à $5 \mathrm{~mm} / 1000$ ans pour une période qui couvre l'Holocène (58 nodules, 1429 mesures). L'étude hydrochimique a fourni une vitesse moyenne d'érosion de $21,4 \mathrm{~mm} / 1000$ ans. Le taux médian de 17,2 mm/1000 ans se rapproche plus de la vitesse réelle d'érosion chimique actuelle, car les mesures ont surtout été prises en été et au printemps. Parmi les nombreux facteurs qui expliquent l'écart entre les taux d'érosion fournis par les deux méthodes, la différence de milieu étudié est primordiale. Elle est mise en évidence par la méthode ubiquiste de l'étude des plaquettes qui a fourni des taux moyens d'érosion presque huit fois plus importants dans les ruisseaux que dans les sols. Dans ces derniers, le résultat obtenu après une expérience de 18 mois a été de 12,4 $\mathrm{mm} / 1000$ ans alors qu'il a été de $94,3 \mathrm{~mm} / 1000$ ans pour les plaquettes immergées dans diverses eaux courantes. Dans les eaux calmes, le résultat a été de $41,7 \mathrm{~mm} / 1000$ ans.
Tous droits réservés @ Les Presses de l'Université de Montréal, 1994

Ce document est protégé par la loi sur le droit d'auteur. L'utilisation des services d’Érudit (y compris la reproduction) est assujettie à sa politique d'utilisation que vous pouvez consulter en ligne.

https://apropos.erudit.org/fr/usagers/politique-dutilisation/ 


\title{
VARIABILITÉ DE L'ÉROSION ACTUELLE ET HOLOCĖNE: LE CAS DES MARBRES DE GRENVILLE EN OUTAOUAIS QUÉBÉCOIS*
}

\author{
Clément PRÉVOST ${ }^{\star *}$ et Bernard LAURIOL, Département de géographie, Université d’Ottawa, Ottawa, Ontario K1N 6 N5.
}

RÉSUMÉ Cet article souligne la difficulté de comparer les taux d'érosion mesurés dans les marbres de Grenville selon différentes méthodes. La variabilité des taux d'érosion a été mise en évidence par l'étude des nodules en relief, des plaquettes de calcaire et par l'hydrochimie. La mesure moyenne du déchaussement postglaciaire des nodules emprisonnés dans les marbres se situe à $5 \mathrm{~mm} / 1000$ ans pour une période qui couvre l'Holocène (58 nodules, 1429 mesures). L'étude hydrochimique a fourni une vitesse moyenne d'érosion de $21,4 \mathrm{~mm} / 1000$ ans. Le taux médian de $17,2 \mathrm{~mm} / 1000$ ans se rapproche plus de la vitesse réelle d'érosion chimique actuelle, car les mesures ont surtout été prises en été et au printemps. Parmi les nombreux facteurs qui expliquent l'écart entre les taux d'érosion fournis par les deux méthodes, la différence de milieu étudié est primordiale. Elle est mise en évidence par la méthode ubiquiste de l'étude des plaquettes qui a fourni des taux moyens d'érosion presque huit fois plus importants dans les ruisseaux que dans les sols. Dans ces derniers, le résultat obtenu après une expérience de 18 mois a été de $12,4 \mathrm{~mm} / 1000$ ans alors qu'il a été de $94,3 \mathrm{~mm} / 1000$ ans pour les plaquettes immergées dans diverses eaux courantes. Dans les eaux calmes, le résultat a été de $41,7 \mathrm{~mm} / 1000$ ans.
ABSTRACT The variability of present-day and Holocene erosion within Grenville marbles on the Québec side of the Ottawa River. This paper demonstrates the difficulties of comparing the variability of erosion rates within Grenville marbles by the use of experimental limestone tablets, the measurement of deterration height of insoluble nodules, and hydrochemical analyses. The mean value of postglacial deterration is $5 \mathrm{~mm} / 1000$ years (58 sites, 1429 measurements). Hydrochemical monitoring yielded a mean erosion rate of $21,4 \mathrm{~mm} / 1000$ years. The median of $17,2 \mathrm{~mm} / 1000$ years likely reflects the actual rate of chemical erosion, as most monitoring took place during spring and summer. There are numerous factors that can account for differences in erosion rates obtained from these two methods, the most predominant one being the site selected. This is evident when the results obtained from experiments using marble tablets are considered. The tablets, when suspended in streams, yielded mean rates of erosion which proved to be almost eight times higher than those measured in soils. Following 18 months of monitoring, the mean rates obtained for tablets in soils and various stream currents were 12,4 and $94,3 \mathrm{~mm} /$ 1000 years respectively. In calm waters, a mean rate of $41,7 \mathrm{~mm} / 1000$ years was calculated.
ZUSAMMENFASSUNG Variabilität der Erosion in der Gegenwart und im Holozän: Der Fall des Marmors von Grenville im Quebeker Outaouais. Dieser Aufsatz hebt hervor, wie schwierig es ist, die im Marmor von Grenville mit verschiedenen Methoden gemessenen Erosionsraten zu vergleichen. Die Variabilität der Erosionsraten konnte durch das Studium der hervorstehenden Knollen, der Kalkplättchen und durch hydrochemische Analysen deutlich gezeigt werden. Der durchschnittliche Wert der postglazialen Freilegung der in den Marmor eingeschlossenen Knollen beträgt $5 \mathrm{~mm} / 1000$ Jahre für die Zeit des Holozän (58 Knollen, 1429 Messungen). Die hydrochemische Analyse ergab eine durchschnittliche Erosionsgeschwindigkeit von $21,4 \mathrm{~mm} / 1000$ Jahre. Die mittlere Rate von 17,2 mm/1000 Jahre kommt der realen gegenwärtigen chemischen Erosionsgeschwindigkeit näher, denn die Messungen wurden vor allem im Sommer und Frühling durchgeführt. Unter den zahlreichen Faktoren, welche die Abweichung zwischen den durch die zwei Methoden erbrachten Erosionsraten erklären, ist der Unterschied des studierten Milieus entscheidend. Er wird deutlich dargelegt durch die allgegenwärtige Methode der Analyse der Plättchen, welche fast acht mal höhere durchschnittliche Erosionsraten in den Flüßchen als in den Böden erbrachte. In den letzteren hat man nach einer 18 monatigen Studie ein Ergebnis von $12,4 \mathrm{~mm} / 1000$ Jahre erhalten, wogegen es $94,3 \mathrm{~mm} / 1000$ Jahre für die in verschiedenen fließenden Wassern eingetauchten Plättchen betrug. In stehenden Gewässern betrug das Ergebnis $41,7 \mathrm{~mm} / 1000$ Jahre.

\footnotetext{
Manuscrit reçu le 10 novembre 1993; manuscrit révisé accepté le 1er mars 1994

* Contribution de la Commission géologique du Canada $n^{\circ} 48193$

** Adresse actuelle: Commission géologique du Canada, 601 rue Booth, Ottawa (Ontario) K1A OE8
} 


\section{INTRODUCTION}

Depuis quelques décennies, notamment depuis les travaux de Corbel (1959a, 1959b), plusieurs spécialistes de la géomorphologie karstique s'efforcent de quantifier les taux d'érosion des terrains calcaires. En Outaouais québécois, la notion de " terrain calcaire " fait référence à un socle carbonaté bien particulier: le marbre de Grenville. Ce marbre abonde dans la province géologique de Grenville, au sud-est du Bouclier précambrien (Wilson, 1925; Thomson, 1956). II a été admis qu'il n'offre pas la physiographie caractéristique des terrains calcaires, puisqu'il affleure principalement dans les zones déprimées du relief (Dresser et Denis, 1946; Mackay, 1949; Ek, 1980-1982). II en résulte que la morphologie karstique n'est pas toujours évidente. Les cavernes, entre autres, n'atteignent jamais de grandes profondeurs, et leur développement horizontal se calcule en quelques centaines de mètres tout au plus. Dans certaines zones, les formes karstiques sont cependant abondantes, ce qui indique que des processus d'érosion actifs et efficaces s'attaquent aux marbres. La quantification de cette érosion a servi de base à une récente étude (Prévost, 1991, 1992). On en présente ici les principaux résultats.

\section{LA RÉGION ÉTUDIÉE}

La région étudiée se situe à une centaine de kilomètres au nord de la ville d'Ottawa, au sud-est du lac Poisson-Blanc (fig. 1). Elle fait partie du plateau Laurentidien ou Laurentides de l'Ouest dont la physiographie a été décrite par Mackay (1947) et Aubert de la Rüe (1948). Cette chaîne de montagnes érodées montre un relief marqué par de nombreux sommets arrondis dont l'altitude varie entre $230 \mathrm{~m}$ et $410 \mathrm{~m}$, et quelques lacs de surcreusement glaciaire occupent les zones basses du relief.

Le socle de la région se compose de roches métasédimentaires d'âge protérozoïque (série de Grenville). Ce sont majoritairement des roches intrusives non différenciées et du calcaire cristallin, avec par endroits des enclaves de gneiss et de quartzite (Ministère des Mines, 1949). La teneur du marbre en carbonates de calcium $\left(\mathrm{CaCO}_{3}\right)$ et de magnésium $\left(\mathrm{MgCO}_{3}\right)$ est généralement supérieure à $95 \%$ (Fréchette, 1916; Goudge, 1935). Du point de vue structural, ce marbre est une roche à grain moyen ou gros, blanche ou grise, renfermant ça et là des nodules composés de feldspaths, de pegmatites, de gabbros et de diorites (Dresser et Denis, 1946).

Le climat est frais et humide. Selon les données des deux stations météorologiques avoisinantes, la température moyenne annuelle est de $4,2^{\circ} \mathrm{C}$. Celle de janvier est de $-12,8^{\circ} \mathrm{C}$ et celle de juillet de $18,8^{\circ} \mathrm{C}$. On compte un peu plus de 160 jours de précipitations par année. La hauteur totale annuelle de ces dernières atteint environ $1000 \mathrm{~mm}$, dont près de $300 \mathrm{~mm}$ provient des chutes de neige (Environnement Canada, 1982). Ces conditions sont favorables au développement d'une forêt mixte, la forêt laurentienne, où conifères et feuillus se côtoient (Hosie, 1979). Cette forêt et son histoire holocène ont récemment été décrites par Richard (1993).

\section{BUT DE L'ÉTUDE}

La recherche vise à montrer que, même à l'intérieur d'une petite région, les taux d'érosion varient selon les milieux et les méthodes utilisées. Un seul type de mesure ne rend pas compte de la variabilité de l'érosion continentale. Pour l'illustrer, on a utilisé trois méthodes classiques en géomorphologie karstique : la mesure de la hauteur du déchaussement de nodules insolubles, celle du taux d'ablation d'un bassin versant établi à partir de mesures hydrochimiques et celle de la perte de masse de plaquettes calcaires laissées sur le terrain pendant une certaine période. Goudie (1981), Trudgill (1983), White (1988), ainsi que Ford et Williams (1989) ont évalué les mérites respectifs de ces méthodes. L'étude veut aussi améliorer la connaissance de la dynamique géomorphologique actuelle et holocène sur un socle cristallin en forêt mixte dans l'est du Canada.

\section{MÉTHODOLOGIE}

\section{NODULES EN RELIEF}

Les mesures du déchaussement des nodules ont été effectuées suivant la méthode décrite par Dahl (1967). Cette méthode a plus récemment été utilisée par d'autres chercheurs, notamment Åkerman (1983) au Spitzberg, ainsi que Dionne et Michaud $(1985,1986)$ sur la côte est de la baie

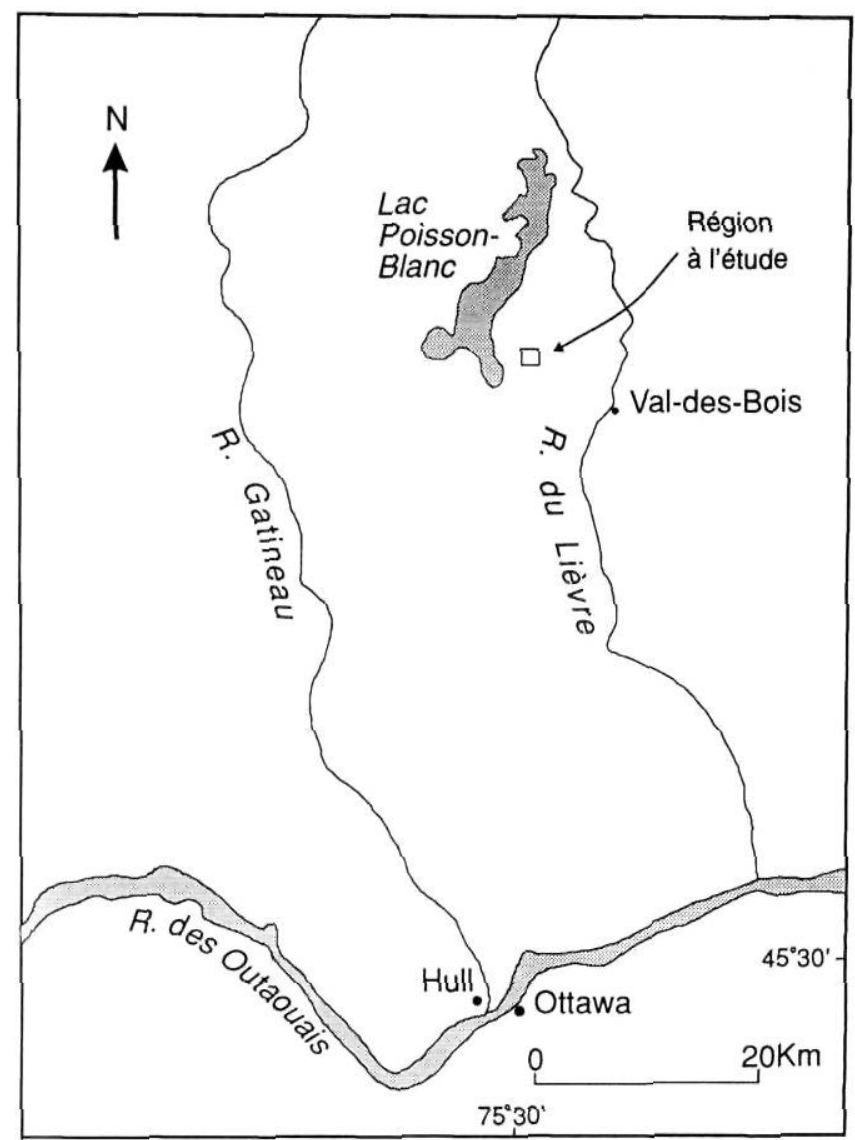

FIGURE 1. Localisation de la région à l'étude.

Location map of the study area. 
d'Hudson. Elle consiste à évaluer la hauteur du déchaussement des nodules insolubles contenus dans la roche mère, puis à diviser ce résultat par une durée. Les 58 nodules retenus ici portaient sur leur face supérieure soit des stries glaciaires (orientées vers $170^{\circ}$ en moyenne), soit un poli glaciaire, preuve de leur affleurement au moment de la déglaciation. Celle-ci est survenue dans la région au tout début de l'Holocène (Dyke et Prest, 1987). La durée de mise en relief des nodules correspond donc à environ 10000 ans. La figure 2 montre bien la ligne de référence qui a permis d'effectuer les mesures de déchaussement. Sur la photo, on distingue nettement la position qu'occupait la surface calcaire au début de l'Holocène. Les surfaces rugueuses du nodule se trouvaient emprisonnées dans le marbre. Une mesure de déchaussement effectuée sur un tel nodule constitue une quantification absolue de l'abaissement postglaciaire de la surface calcaire. Les nodules qui ne présentaient pas cette nette démarcation ont été rejetés.

Afin d'assurer un niveau de comparaison valable entre les différents sites, on a retenu plusieurs types de mesures. En plus de la hauteur du déchaussement, on a mesuré l'exposition du site du nodule, sa surface d'exposition et la pente de la surface sur laquelle il repose. La valeur du déchaussement a été mesurée au millimètre près à l'aide d'un ruban de vinyle. De dix à cinquante mesures de hauteur ont été faites autour de chaque nodule. La pente de la surface calcaire a été déterminée à l'aide d'un clinomètre à lecture directe Suunto à précision de $\pm 0,5^{\circ}$. L'exposition de l'affleurement du site a été mesurée en utilisant une boussole Sylva. Comme le marbre de Grenville présente de nombreuses variations de faciès, on a prélevé à chacun des sites des fragments de la roche à l'intérieur de laquelle les nodules étaient inclus. Leur teneur en carbonates a été déterminée par une analyse Chittick (Dreimanis, 1962).

Plus de 1400 mesures de déchaussement ont fait l'objet d'un traitement informatique via le système d'analyse statistique SAS (Helwig, 1983). On a tenté de trouver, à l'aide de la régression multiple, un modèle linéaire généralisé permettant de connaître le poids explicatif des variables surface, pente, orientation et teneur en carbonates sur la variable déchaussement. On a donc mis en évidence le degré d'explication de chacune des variables indépendantes vis-à-vis du déchaussement des nodules, variable dépendante.

\section{HYDROCHIMIE}

L'approche hydrochimique proposée se base sur les principes méthodologiques conventionnellement admis (Goudie, 1981; Trudgill, 1983, 1985; White, 1988; Stednick, 1991). Elle consiste à mesurer la minéralisation, le $\mathrm{pH}$, la température et le débit en un point qui permet de caractériser la vitesse de dissolution à l'intérieur d'un bassin versant.

On a effectué l'échantillonnage à l'embouchure d'un ruisseau qui draine un bassin versant boisé de $1,7 \mathrm{~km}^{2}$. Le volume d'eau a été déterminé selon la méthode de Schoeller (1962). Pour convertir la vitesse de surface en vitesse de profondeur, on a pris soin d'appliquer le coefficient de frottement proposé par Gardiner et Dackombe (1983). Outre l'analyse de l'eau issue du ruisseau, une vingtaine d'analyses de miné-

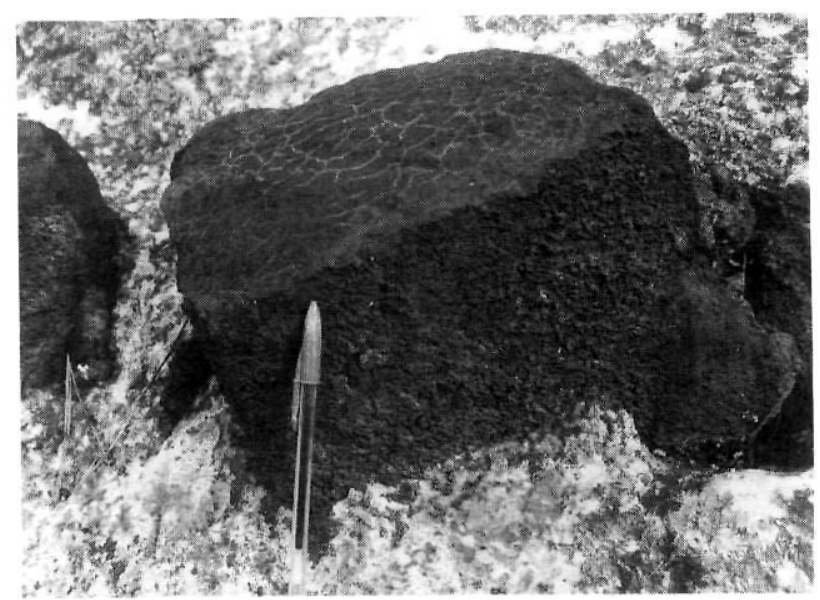

FIGURE 2. Un nodule en relief, région du lac Sainte-Marie, vallée de la rivière Gatineau, Québec.

Nodule in relief, Lac Sainte-Marie area, Rivière Gatineau valley, Québec.

ralisation ont été effectuées sur des eaux météoriques (pluie et neige fondante), ainsi que sur des échantillons de neige fraîchement tombée au sol (Prévost, 1991). La formule utilisée pour effectuer les calculs de dénudation chimique est celle proposée par Groom et Williams (1965). Elle est aussi décrite de façon détaillée par Chambers (1983):

$$
X=(E-A) /\left(D \times S \times 10^{6}\right) \quad \text { Équation } 1
$$

où $X$ représente la dénudation karstique exprimée en $\mathrm{mm} /$ millénaire, E les éléments dissous évacués du bassin par le ruisseau en g/an, A l'apport allochtone via les précipitations en $\mathrm{g} / \mathrm{an}, \mathrm{D}$ la densité du marbre en $\mathrm{g} / \mathrm{cm}^{3}$, et $\mathrm{S}$ la surface du bassin de drainage en $\mathrm{km}^{2}$.

Les mesures de minéralisation ont été effectuées dans des ruisseaux. Les paramètres hydrologiques et hydrochimiques ont été mesurés particulièrement au printemps, car c'est la saison où ceux-ci sont susceptibles de changer rapidement en raison de la fonte de la neige et de la reprise de la vie végétative.

\section{PLAQUETTES DE MARBRE}

La méthode des plaquettes demeure fort populaire auprès des chercheurs œuvrant en milieu karstique. Tout d'abord mise en application par Chevalier (1953) et Gams (1959), elle a depuis été raffinée et utilisée au cours de bon nombre de recherches (entre autres: Kupper et Pissart, 1974; Kupper, 1985; Gérome-Kupper, 1984; Bocquet, 1985; Droppa, 1985; Ugarte et Ugalde, 1985; Urushibara-Yoshino, 1993). Le principe de base de la méthode consiste à installer pour un temps donné des morceaux de calcaire (ici de marbre) dans des endroits représentatifs, de telle sorte que les agents d'érosion atteignent les échantillons. Les plaquettes doivent être pesées avant et après leur séjour sur le terrain et toute perte de masse est attribuable à l'action de ces agents d'érosion (Nicod, 1983).

Quarante plaquettes de marbre ont été sciées dans deux blocs sains recueillis près d'une caverne de la région. On a choisi d'employer un matériel local de façon à avoir des 


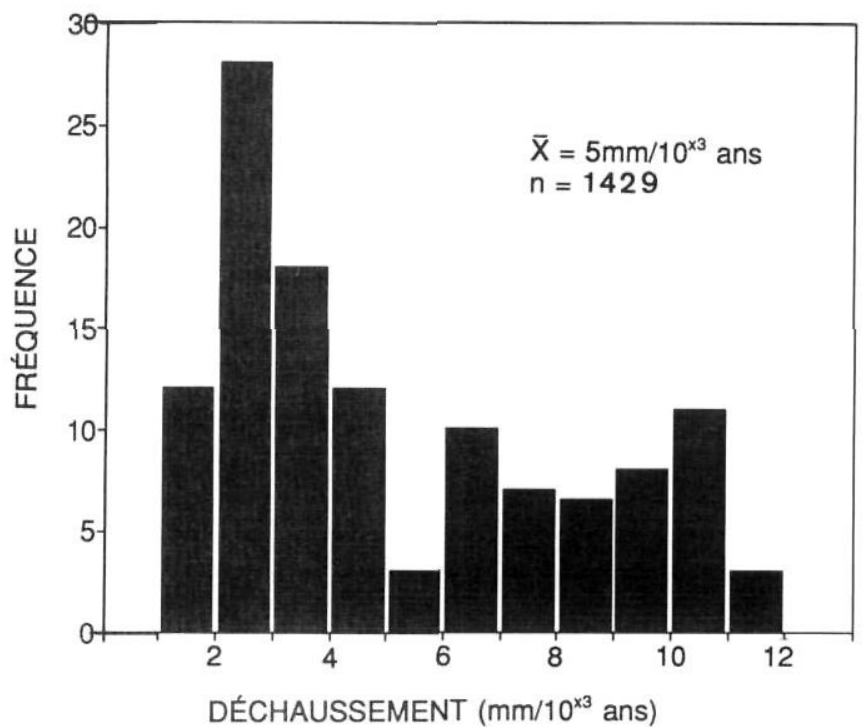

FIGURE 3. Fréquence relative des hauteurs de déchaussement des nodules.

Relative frequency of deterration heights of nodules.

paramètres d'expérimentation se rapprochant le plus possible des conditions de terrain. Egalement, Crowther (1983) et Bocquet (1985) signalent qu'en utilisant des plaquettes provenant d'un matériel local, on est en mesure de déterminer des valeurs absolues de dénudation karstique. On a séparé les plaquettes en deux groupes (1 et 2) de vingt unités. Les dimensions ont été mesurées au millimètre près. Les plaquettes du groupe 1 avaient en moyenne $4,7 \mathrm{~cm}$ de longueur, $4,5 \mathrm{~cm}$ de largeur et $0,9 \mathrm{~cm}$ d'épaisseur. Les plaquettes du groupe 2 avaient des dimensions de $4,5 \times 4,1 \times$ $2 \mathrm{~cm}$. Les pesées ont été faites à $0,001 \mathrm{~g}$ près à l'aide d'une balance électronique Mettler-H4, après nettoyage et séchage des échantillons.

Les plaquettes ont été disposées sur le terrain par groupe de deux (une de chaque groupe), soit enfouies dans le sol ou bien immergées dans des eaux courantes ou calmes. En milieu aquatique, on a suspendu les échantillons à l'aide d'une broche de laiton rattachée à une cordelette entourant les plaquettes, afin qu'elles soient totalement immergées, mais disposées de façon à éliminer les frottements. En milieu terrestre, on a enfoui les plaquettes à une quinzaine de centimètres de profondeur dans différents types de sols. La cueillette des échantillons pour pesage s'est effectuée après des périodes de six mois environ. Bocquet (1985) a constaté que cette durée d'exposition semblait optimale pour bien apprécier les variations au niveau de l'érosion.

L'expérience a duré au total 542 jours. Pour transformer la perte de masse des plaquettes en hauteur de dénudation karstique $(\mathrm{H})$, l'équation suivante a été retenue (Trudgill, 1975, 1983, 1985) :

$$
\mathrm{H}=(\mathrm{P} / \mathrm{D} / \mathrm{S}) \times 10^{4} \quad \text { Équation } 2
$$

où $\mathrm{P}$ est la perte annuelle de masse en $\mathrm{g} / \mathrm{an}$, $\mathrm{D}$ la densité de la plaquette en $\mathrm{g} / \mathrm{cm}^{3}$, et $\mathrm{S}$ la surface de cette dernière en $\mathrm{cm}^{2}$. Le multiplicateur permet de transformer les unités originales de $\mathrm{H}$ (cm/an) en $\mathrm{mm} / 1000$ ans.

\section{NOTATION BUBNOFF}

La dénudation karstique s'exprime soit en volume annuel de roche $\left(\mathrm{m}^{3} / \mathrm{km}^{2} / \mathrm{an}\right)$, soit par l'épaisseur d'une couche calcaire qui aurait été uniformément enlevée de toute la surface d'un bassin ( $\mathrm{mm} / 1000$ ans). Un $\mathrm{m}^{3} / \mathrm{km}^{2} /$ an équivaut à un $\mathrm{mm} / 1000$ ans (Hakim, 1984; Ford et Williams, 1989). L'unité $\mathrm{mm} / 1000$ ans correspond aussi à la notation Bubnoff, symbolisée B, telle que décrite par Fischer (1969). Cette notation peut s'appliquer autant à la quantification du soulèvement isostatique qu'à celle de l'érosion (Ollier, 1981). Cette dernière notion est toutefois purement théorique, puisque l'érosion se répartit toujours très inégalement, même sur socle calcaire (Hakim, 1984). Ce chiffre mesurant l'épaisseur moyenne de roche évacuée s'utilise par convention et il a le net avantage de permettre des comparaisons entre divers sites et via diverses méthodes (Ford et Williams, 1989). Pour alléger le texte, on attribuera ici aux valeurs de dénudation karstique le symbole B (unité Bubnoff).

\section{RÉSULTATS}

\section{NODULES EN RELIEF}

Un total de 1429 mesures de déchaussement ont été effectuées autour de 58 nodules. Les valeurs de déchaussement s'échelonnent entre 12 et $121 \mathrm{~mm}$ (fig. 3). La hauteur moyenne se situe à $49 \mathrm{~mm}$ (écart type de 5,7 $\mathrm{mm}$ ). En répartissant le déchaussement sur toute la période postglaciaire (environ $10^{4}$ ans), on obtient une dénudation karstique moyenne de $5 \mathrm{~B}$. La différence moyenne entre le déchaussement médian et le déchaussement moyen s'établit à seulement 1,2 mm. L'influence des divers facteurs qui expliquent le déchaussement est évaluée par l'équation suivante:

$H=3,18 C+0,03 S-6,12 R+11,42 P-236,49$ Équation 3 où $\mathrm{H}$ est la hauteur du déchaussement en $\mathrm{mm}, \mathrm{C}$ la teneur en $\mathrm{CaCO}_{3}$ du marbre encaissant, $\mathrm{S}$ la surface exposée du nodule en $\mathrm{cm}^{2}$, R le cosinus de l'orientation du site et $\mathrm{P}$ le cosinus de la pente de l'affleurement rocheux. Ce modèle explique plus de $61 \%$ du déchaussement et il indique que $42 \%$ de celui-ci est attribuable au degré de pureté du marbre (teneur en carbonates). Les autres variables ont un poids explicatif plus faible: la pente de la roche sur laquelle le nodule émerge représente $7 \%$, l'exposition du site $2 \%$ et la surface du nodule $9 \%$. Il est évident que l'introduction d'autres variables changerait les résultats, puisque près de $39 \%$ des variations sont inexpliquées. L'importance du rôle tenu par la lithologie doit toutefois être souligné. Elle rend compte du fait que certains nodules ont une hauteur de $12 \mathrm{~cm}$ alors que d'autres dépassent à peine $1 \mathrm{~cm}$.

Plus de $90 \%$ des nodules montrent une distribution bimodale de hauteur: un côté est plus en relief que l'autre (fig. 4). Une observation semblable a été faite par Dahl (1967), en Norvège, sur des veines de quartz mises en relief. II n'a toutefois trouvé une distribution bimodale que dans $26 \%$ des cas. Dans les cas extrêmes en Outaouais, la différence de déchaussement a atteint près de $50 \mathrm{~mm}$. Dans $83 \%$ des cas, le déchaussement s'est avéré plus important en aval qu'en amont du nodule (bas de pente vs haut de pente). Pour expliquer ce phénomène, on ne peut qu'émettre des hypothèses. 
FIGURE 4. Distribution des hauteurs de déchaussement d'un nodule. Les mesures ont été prises à tous les centimètres autour du nodule.

Distribution of deterration heights around a nodule. Measurements were taken every centimetre.

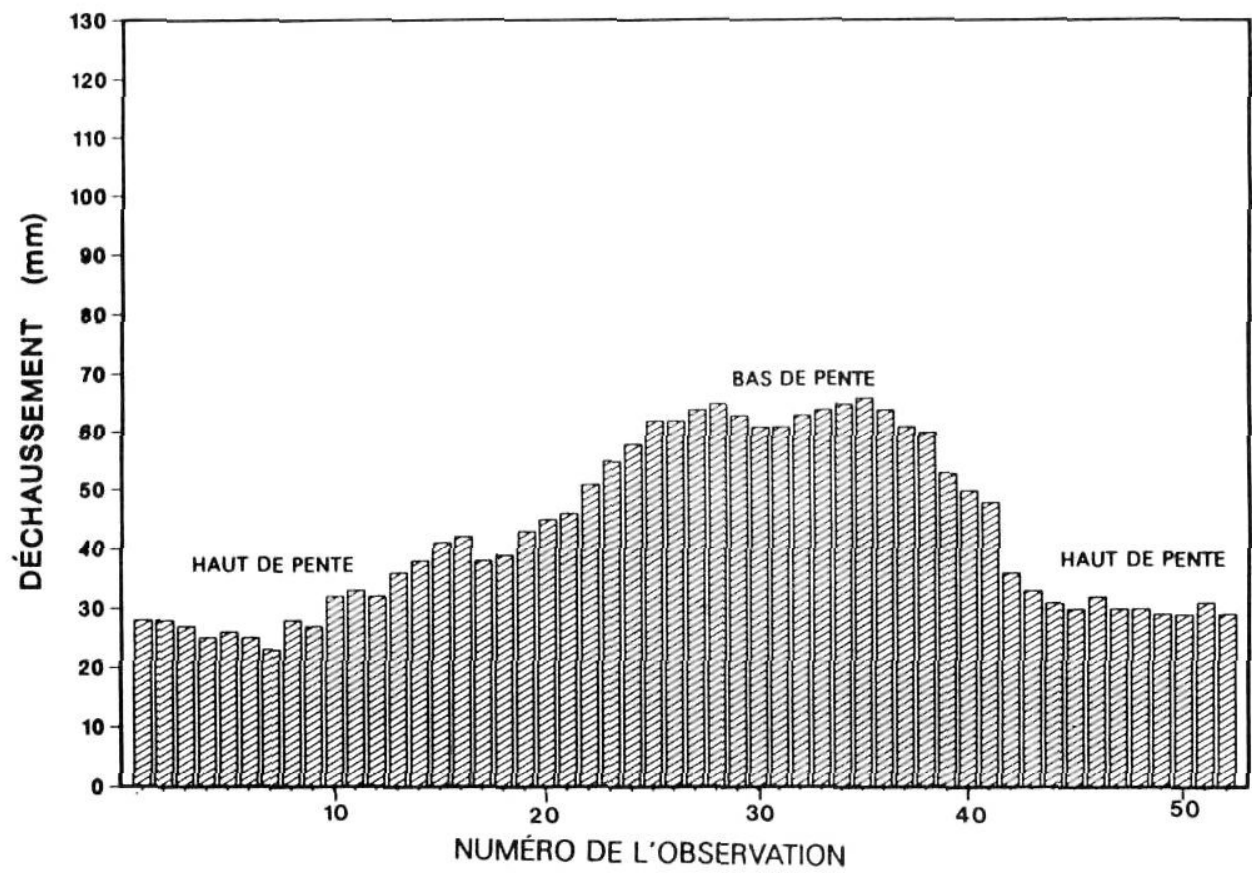

L'acidification de l'eau de ruissellement lors de son contact avec le nodule semble à ce jour la plus plausible. Par suite de cette acidification, l'eau attaque davantage le côté aval du nodule. Reddy (1988) a en effet montré que l'agressivité de l'eau change rapidement suivant la lithologie sur laquelle elle ruisselle. Or, on sait que les nodules contiennent du mica, du feldspath, des pegmatites, du gneiss, des gabbros, etc. (Dresser et Denis, 1946). Ils sont donc nettement plus acides que le calcaire cristallin encaissant. En deuxième lieu, la concentration de l'humidité du côté aval des nodules pourrait aussi faciliter ce travail de dissolution différentielle.

Les résultats acquis sont les premiers à quantifier l'abaissement postglaciaire de la surface calcaire dans les marbres de Grenville. Ils se comparent avec les taux obtenus ailleurs par d'autres chercheurs. Sur substrat granitique en Norvège, Dahl (1967) a calculé un abaissement postglaciaire de la surface de près de 1,2 $\mathrm{B}$ à l'aide de la méthode des nodules et des veines en relief. Au Spitsberg, Åkerman (1983) a mesuré le déchaussement des veines et des nodules émergeant d'une surface dolomitique. II a obtenu un abaissement moyen de 2,5 B. Également par la méthode des nodules en relief, Dionne et Michaud (1986) ont évalué à près de 6 B l'abaissement d'un substrat de dolomie aux abords du lac Guillaume-Delisle, au sud-est de la baie d'Hudson. Le taux moyen de près de $5 \mathrm{~B}$ mesuré pour la région étudiée s'inscrit dans un ordre de grandeur très plausible. Les différences de déchaussement, d'une région à l'autre ou à l'intérieur d'une même région, ne peuvent cependant être expliquées seulement par des facteurs climatiques, comme l'ont indiqué Dionne et Michaud (1986). Le modèle linéaire développé ici montre bien que les différences lithologiques introduisent des variations considérables dans les vitesses d'érosion.

\section{HYDROCHIMIE}

Entre décembre 1987 et mai 1989, on a effectué environ 250 analyses hydrochimiques des eaux de la région étudiée
(Prévost, 1991). En dix-huit occasions, on a aussi mesuré le débit de l'eau au même site. Les taux de dénudation qui en ont été déduits s'échelonnent entre 12,7 et 49,3 B. La valeur moyenne s'établit à 21,4 B et la médiane se chiffre à 17,2 B. Les valeurs les plus fortes s'observent au printemps où elles se situent entre 25 et près de $50 \mathrm{~B}$. Les plus faibles valeurs coïncident avec la période estivale : du 12 juin au 24 septembre 1988, la dénudation a affiché des valeurs minimale et maximale de 12,7 et $19 \mathrm{~B}$. Le facteur responsable de cette situation est la baisse du débit. Le débit et la quantité d'éléments dissous évacués sont deux variables proportionnellement reliées ( $E k, 1969)$. Une baisse du débit provoque une baisse incidente de la charge transportée, même si les concentrations en magnésium et en calcium augmentent. Au printemps, les faibles concentrations sont liées à un effet de dilution occasionné par les forts débits attribuables aux crues printanières et à une faible disponibilité du gaz carbonique. Les concentrations de $\mathrm{CO}_{2}$ augmentent d'ailleurs très rapidement dans la forêt boréale quand les arbres se couvrent de feuilles (Lauriol et al., 1987).

Les résultats précédents ne s'appuient que sur un nombre restreint de données. On ne dispose en effet que de trois valeurs pour caractériser la période s'étalant entre le 10 octobre et le 5 mai. On est tout de même en mesure de croire que les valeurs de dénudation karstique hivernale auraient été semblables à celles calculées pour la période comprise entre le 5 juin et le 24 septembre. En effet, c'est durant les mois d'hiver qu'on a enregistré les plus hautes valeurs de dureté totale, avec des concentrations moyennes d'environ $60 \mathrm{mg} / \mathrm{l}$ $\mathrm{CaCO}_{3}$ (Prévost, 1991). Également, le débit à cette période de l'année est aussi bas que durant la saison estivale. Pour cette période estivale, la moyenne de dénudation chimique se chiffrait à 15,1 B. Comme les valeurs printanières élevées contribuent à hausser la moyenne de dénudation chimique, on croit que la valeur médiane de 17,2 B se rapproche plus de l'intensité réelle de l'érosion. 


\section{PLAQUETTES DE MARBRE}

Les résultats relatifs aux plaquettes de marbre sont compilés à l'intérieur du tableau I. Les plaquettes enfouies dans les sols ont fourni des taux de dénudation qui varient entre 2,5 et 27,3 B pour une moyenne de 12,4 B (médiane de 11,9 B). Le phénomène le plus évident est d'abord la différence entre les plaquettes du groupe 1 et celles du groupe 2 . Les plaquettes du groupe 1, plus minces, ont fourni les valeurs de dénudation les plus élevées (moyenne $=16,1 \mathrm{~B}$ ). Les plaquettes du groupe 2 ont offert une dénudation moyenne de $8,7 \mathrm{~B}$. Ce phénomène s'explique par le fait que le calcul du taux de dénudation fait intervenir la masse et la surface : une plaquette offrant une grande surface et une faible masse est plus sensible à l'érosion qu'une plaquette ayant la même surface, mais une plus grande masse (Bocquet, 1985). La même situation s'observe au niveau des plaquettes suspendues dans les divers milieux aquatiques. En eaux calmes, les plaquettes minces du groupe 1 ont fourni un taux moyen de dénudation de 68,4 B. Ce taux est presque cinq fois plus élevé que celui des plaquettes du groupe 2 (15 B). Dans les eaux courantes, les différences sont encore plus frappantes et elles vont dans le même sens (groupe 1 $=161,8 \mathrm{~B}$; groupe $2=26,7 \mathrm{~B}$ ).

En considérant individuellement chaque groupe de plaquettes, on est en mesure de constater à quel point l'érosion varie d'un milieu à l'autre. Par exemple, au niveau des plaquettes minces du groupe 1 , on remarque que le taux moyen d'érosion est multiplié par dix lorsqu'on passe des sols aux eaux courantes (sols $=16,1 \mathrm{~B}$; eaux calmes $=68,4 \mathrm{~B}$; eaux courantes $=161,8 \mathrm{~B}$ ). Des différences moins grandes s'observent sur les plaquettes plus épaisses du groupe 2, mais la tendance est la même (sols $=8,7 \mathrm{~B}$; eaux calmes $=15 \mathrm{~B}$; eaux courantes $=26,7 \mathrm{~B}$ ). L'explication des variations d'érosion d'un milieu à l'autre est assez bien connue dans ses grandes lignes. Plusieurs recherches ont montré que la vitesse d'érosion des plaquettes est directement reliée à la vitesse d'écoulement de l'eau (Roques et Ek, 1973; Kupper, 1979, 1985; Morel et al., 1978-79). Une même vitesse de l'eau ne donne toutefois pas nécessairement lieu à une même vitesse d'érosion, puisqu'une ou plusieurs autres variables entrent en jeu. L'érosion des plaquettes déposées dans les sols dépend surtout de la quantité d'eau reçue. Ainsi, dans le groupe 1, la plaquette qui a perdu le moins de masse (10,1 B) était située dans le sol sous une dense touffe de thuyas, lesquels interceptent les chutes de neige et la pluie. Dans ce même groupe, la plaquette qui a montré la valeur de dénudation la plus élevée $(27,3$ B) avait été déposée au fond d'une petite doline en milieu ouvert. On note les mêmes faits avec les plaquettes du groupe 2 (sous un lit de mousse $=2,5 \mathrm{~B}$; au fond d'une doline $=11,8 \mathrm{~B}$ ).

\section{DISCUSSION}

Les résultats présentés ci-dessus montrent bien que les taux d'érosion peuvent varier de façon considérable à l'intérieur d'une même région. Ces variations sont principalement introduites par le choix des sites et des méthodes d'analyse. II est par conséquent peu conseillé de comparer d'une façon absolue les taux de dénudation fournis par les plaquettes à
TABLEAU ।

Hauteurs de dénudation karstique, en notation Bubnoff, déterminées par la méthode des plaquettes calcaires (région du lac Poisson-Blanc)

\begin{tabular}{lrcc}
\hline & Sols & Eaux calmes & Eaux courantes \\
\hline Goupe 1 & 16,1 & 68,4 & 161,8 \\
Groupe 2 & 8,7 & 15,0 & 26,7 \\
Moyenne & 12,4 & 41,7 & 94,3 \\
\hline
\end{tabular}

ceux obtenus de la mesure de déchaussement des nodules ou de la minéralisation des eaux. Dans le cas des nodules en relief par exemple, l'érosion n'agit que sur un seul plan de surface. De plus, l'apport en eau est toujours très limité. L'eau n'est en effet disponible qu'au moment de la fonte nivale ou lors des périodes de pluie, soit durant environ 60 à 70 jours par an (Environnement Canada, 1982). Le reste du temps, les nodules sont soit recouverts de neige, soit en milieu sec. Dans le cas des plaquettes, l'érosion agit sur plusieurs surfaces fraîchement mises à nu à la suite du sciage des échantillons. En milieu aquatique, l'abrasion joue sûrement un rôle prépondérant dans l'érosion des plaquettes de calcaire. Dans les cours d'eau, l'usure mécanique provoquée par les impacts d'éléments en suspension peut en effet prendre une importance assez significative (Trudgill, 1970, 1983; Bocquet, 1985). L'intérêt des plaquettes est en fait de mettre en évidence le potentiel d'altération au sein des divers milieux représentés (Crowther, 1983; Trudgill, 1983). En ce sens, une différence assez nette apparaît entre les trois milieux dans lesquels on a déposé les échantillons (tabl. I).

Le déchaussement des nodules a fourni le taux moyen d'érosion le plus faible dans le cadre de l'étude, soit $5 \mathrm{~B}$. L'hydrochimie a fourni un taux moyen de plus de $21 \mathrm{~B}$. La différence s'explique par le fait que les deux milieux étudiés et les deux méthodes utilisées sont très différents : les nodules sont situés sur des surfaces rocheuses qui sont sèches pendant une partie de l'année et ils sont déchaussés par l'érosion aérolaire et l'activité biologique (Viles, 1984). Au contraire, l'hydrochimie mesure le matériel transporté au moment où les eaux s'écoulent et passent en un point donné d'un bassin versant. À l'intérieur de celui-ci cependant, l'eau érode plus ou moins activement. La méthode des plaquettes montre quelques-unes de ces variations, notamment que l'érosion est beaucoup plus forte dans les ruisseaux que dans les sols. À l'intérieur des cavernes, des nodules en relief illustrent également bien ce phénomène. Dans l'Outaouais, plusieurs cavernes actives exhibent des nodules de 20 à $30 \mathrm{~cm}$ de hauteur. Au-delà de cette hauteur, ils se détachent des parois et sont entraînés par le courant. Comme leur surface n'est pas datable, à la différence des nodules extérieurs, on ne peut en déduire le taux de dénudation. Mais le simple fait qu'ils montrent des hauteurs plus importantes qu'en situation extérieure indique déjà une érosion plus active.

\section{CONCLUSION}

On constate donc que l'étude des taux de dénudation karstique en milieu naturel demeure une tâche complexe. Le 
problème consiste à transformer des valeurs ponctuelles acquises dans une courte période en valeurs représentatives d'une période beaucoup plus longue. En fait, les données ponctuelles de dénudation karstique peuvent difficilement définir un espace et un temps plus important que ce qu'elles caractérisent véritablement, comme le notent Ford et Williams (1989). À l'intérieur d'une même région, le taux d'érosion varie suivant les divers paramètres naturels, les méthodes employées et le nombre d'échantillons en présence. II est donc difficile de comparer les résultats acquis à l'intérieur d'une région à ceux obtenus ailleurs. La comparaison est d'autant plus difficile lorsque les mesures ont été effectuées à l'aide de méthodes diverses. II faut admettre qu'en exprimant les résultats en unités Bubnoff, la comparaison est tentante. On ne doit cependant pas perdre de vue qu'elle demeure risquée.

\section{REMERCIEMENTS}

Les auteurs remercient MM. Jacques Dubuc et Daniel St-Jean, respectivement propriétaires des pourvoiries Pointe-des-Pins et Chevreuil-Blanc. Sans leur coopération, aucune donnée n'aurait pu être recueillie. À quelques reprises, Manon Desforges a fourni une aide précieuse sur le terrain. Sheridan Hipwell a bien voulu traduire le résumé et la légende des figures. Les commentaires critiques de $\mathrm{M}^{\text {me }}$ Marie-Anne Geurts, de M. Jacques Schroeder, ainsi que d'un lecteur anonyme ont contribué à l'amélioration du manuscrit. Le premier auteur a bénéficié d'une bourse d'entrée de l'école des Études supérieures et de la Recherche de l'Université d'Ottawa, ainsi que d'une bourse FCAR.

\section{RÉFÉRENCES}

Ákerman, J.H., 1983. Note on chemical weathering, Kapp Linné, Spitsbergen. Proceedings of the $4^{\text {th }}$ International Conference on Permafrost Washington (D.C.), National Academy Press, 10-15.

Aubert de la Rüe, E., 1948. Les caractères physiques des Laurentides de l'Ouest. Bulletin de l'Association des géographes français, 192-193: $34-40$

Bocquet, F., 1985. Dynamique karstique d'un haut massif alpin : la Chaîne des Aravis. Méthodologie et comparaisons avec d'autres massifs montagneux. Annales de la Société géologique de Belgique, 108: 203-207.

Chambers, W., 1983. Denudation rates in the River Burry catchments, Gower, Glamorgan. Cave Science, 10(4) : 181-187.

Chevalier, P., 1953. Erosion or corrosion? Proceedings of the International Speleological Conference of Paris, $1: 35-40$.

Corbel, J., 1959a. Érosion en terrain calcaire (vitesse d'érosion et morphologie). Annales de Géographie, 68(366) : 97-120.

1959b. Vitesse de l'érosion. Zeitschrift für Geomorphologie, 3 (1) $1-29$.

Crowther, J., 1983. A comparaison of the rock tablet and water hardness methods for determining chemical erosion rates on karst surfaces. Zeitschrift für Geomorphologie, 27(1) : 55-64.

Dahl, R., 1967. Post-glacial micro-weathering of bedrock surfaces in the Narvik district of Norway. Geografiska Annaler, 49A(2-4) : 155-166.

Dionne, J.-C. et Michaud, Y., 1985. Observations sommaires sur l'altération chimique au golfe de Guillaume-Delisle. Hudsonie. Annales de l'ACFAS, $52-53: 175$.

1986. Note sur l'altération chimique en milieu périglaciaire, Hudsonie. Québec subarctique. Revue de Géomorphologie dynamique, 35(3): 81-92.
Dreimanis, A., 1962. Quantitative gasometric determination of calcite and dolomite by using Chittick apparatus. Journal of Sedimentary Petrology, 32(3) : 520-529.

Dresser, J.A. et Denis, T.C., 1946. La géologie de Québec, géologie descriptive. Ministère des Mines, Québec, Rapport géologique 20(2), 647 p.

Droppa, A., 1985. Quelques expériences de mesures de la corrosion dans le karst de Demanova (Carpathes Occidentales), Tchécoslovaquie. Annales de la Société géologique de Belgique, 108 : 209-212.

Dyke, A.S. et Prest, V.K., 1987. Late Wisconsinan and Holocene retreat of the Laurentide Ice Sheet. Géographie physique et Quaternaire, 41(2): 237-263.

Ek, C., 1969. Facteurs, processus et morphologie karstiques dans les calcaires paléozoiques de la Belgique. Thèse de doctorat, Université de Liège, $476 \mathrm{p}$.

-1980-82. Les phénomènes karstiques au Québec. Spéléo-Québec, $7-8-9: 5-22$.

Environnement Canada, 1982. Normales climatiques au Canada, température et précipitations, 1951-1980, Québec. Service de l'environnement atmosphérique, Environnement Canada, Publication du Programme climatique canadien, $5:$ ix $+216 p$.

Fisher, A.G., 1969. Geological time-distance rates: The Bubnoff unit. Geological Society of America Bulletin, $80:$ 549-552.

Ford, D.C. et Williams, P.W., 1989. Karst geomorphology and hydrology. Unwin Hyman, London, Boston, $601 \mathrm{p}$.

Fréchette, H., 1916. Pierres calcaires de la Province de Québec. Ministère des Mines, Division des Mines, rapport sommaire 1914 : 36-55.

Gams, I., 1959. Experiment with tablets in the Podpeska cave. Nase Jame, 2 : 76-77.

Gardiner, V. et Dackombe, R.V., 1983. Geomorphological field manual. George Allen and Unwin, Boston, $254 \mathrm{p}$.

Gérome-Kupper, M., 1984. L'érosion des calcaires à l'air libre : mesure de processus actuels. Zeitschrift für Geomorphologie, Suppl. Bd. 49 : 59-74.

Goudge, M.F., 1935. Les calcaires du Canada, partie III (Québec). Commission géologique du Canada, Division des Mines, publication 758.

Goudie, A.S., 1981. Geomorphological techniques. George Allen and Unwin, London, p. 196-222.

Groom, G.E. et Williams, V.H., 1965. The solution of limestone in South Wales. Geographical Journal, 131(1) : 37-41.

Hakim, B., 1984. Nouvelle approche pour l'évaluation de la dissolution spécifique. Karstologia, $4: 56$.

Helwig, J.T., 1983. Guide d'introduction à SAS. SAS Institute Inc., Cary, North Carolina, $94 \mathrm{p}$.

Hosie, R.C., 1979. Arbres indigènes du Canada. Fides (Montréal) et Approvisionnements et Services Canada (Ottawa), $8^{\mathrm{e}}$ éd., $389 \mathrm{p}$.

Kupper, M., 1979. Mesure de l'ablation du calcaire dans une rivière belge. Annales de la Société géologique de Belgique, 102 : 69-70.

— 1985. Les vitesses d'érosion du calcaire dans l'eau : étude des phénomènes actuels. Annales de la Société géologique de Belgique, 108: 261-265.

Kupper, M. et Pissart, A., 1974. Vitesse d'érosion en Belgique de calcaires d'âge Primaire exposés à l'air libre ou soumis à l'action de l'eau courante. Abhandlungen der Akademie des Wissenschaften in Gottingen, 29 : 39-50.

Lauriol, B., Roberge, J. et St-Pierre, L., 1987. Les relations entre la géomorphologie, le climat et l'hydrochimie dans la caverne à la Patate, île d'Anticosti, Canada. Zeitschrift für Geomorphologie, 31(4) : 419-431.

Mackay, J.R., 1947. The north shore of the Ottawa River, Quyon to Montebello, Québec. Revue canadienne de Géographie, 1(2-3) : 3-9.

- 1949. Physiography of the Lower Ottawa Valley. Revue canadienne de Géographie. 3(1-4) : 53-97.

Ministère des Mines, 1949. Région de Val-des-Bois, comtés de Papineau et Gatineau, Québec. Carte géologique préliminaire 697. 
Morel, R., Kupper, M. et Ek, C., 1978-79. Vitesse de dissolution du calcaire sous l'effet de la fonte de la neige : expérience en milieu naturel. SpéléoQuébec, 5-6: 12-25.

Nicod, J., 1983. Nouvelles recherches géomorphologiques sur les karsts méditerranéens. Karstologia, $1: 33-38$.

Ollier, C.D., 1981. Tectonics and landforms. Geomorphology Texts 6 Longman, New York, $214 \mathrm{p}$.

Prévost, C.L., 1991. Processus et intensité actuelle et holocène de l'érosion du marbre de Grenville, région Gatineau-Lièvre, Québec. Thèse de maîtrise, Département de Géographie, Université d'Ottawa, xvii +250 p.

- 1992. Évaluation de la vitesse d'érosion du marbre de Grenville, région Gatineau-Lièvre, Québec. Bulletin de I'AQQUA, 18(1) : 39.

Reddy, M.M., 1988. Acid rain damage to carbonate stone: A quantitative assessment based on the aqueous geochemistry of rainfall runoff from stone. Earth Surface Processes and Landforms, 13(4) : 335-354.

Richard, P.J.H., 1993. Origine et dynamique postglaciaire de la forêt mixte au Québec. Review of Palaeobotany and Palynology, 79 : 31-68.

Roques, H. et Ek, C., 1973. Étude expérimentale de la dissolution des calcaires par une eau chargée de $\mathrm{CO}_{2}$. Annales de Spéléologie, 28(4) : 549-563.

Schoeller, H., 1962. Les eaux souterraines. Masson, Paris, 642 p.

Stednick, J.D., 1991. Wildland water quality. Sampling and analysis. Academic Press, San Diego, $217 p$
Thomson, J.E., 1956. The Grenville problem. Royal Society of Canada, Special Publication 1, $119 \mathrm{p}$.

Trudgill, S.T., 1970. Micro-erosion measurement of exposed bedrock. Area, 2(3) : 61.

- 1975. Measurement of erosional weight-loss of rock tablets. British Geomorphological Research Group, Technical Bulletin, $17:$ 13-19.

1983. Weathering and erosion. Sources and Methods in Geography. Butterworths, London, Boston, $192 \mathrm{p}$.

1985. Limestone geomorphology. Geomorphology Texts 8, Longman, New York, $196 \mathrm{p}$.

Ugarte, F.M. et Ugalde, T., 1985. La dynamique karstique actuelle à AralarMendi (Moyenne Montagne Basco-Cantabrique). Annales de la Société géologique de Belgique, 108 : 221-224.

Urushibara-Yoshino, K., 1993. Solution rate of limestone tablets in Japan Proceedings of the Third International Congress of Geomorphology, McMaster University, Hamilton, abstract, p. 264.

Viles, H., 1984. Biokarst: Review and prospects. Progress in Physical Geography, 8 (4) : 523-542.

White, W.B., 1988. Geomorphology and hydrology of karst terrains. Oxford University Press, New York, $464 \mathrm{p}$.

Wilson, M.E., 1925. The Grenville Pre-Cambrian sub-province. Journal of Geology, 33(4) : 389-407. 